\title{
Report on pilot study of effects of DDT on maternal behavior
}

\author{
KAREN PAULSEN, VINCENT J. ADESSO \\ and \\ JOHN J. PORTER \\ University of Wisconsin, Milwaukee, Wis. 53201
}

To examine the hypothesis that DDT would have an adverse effect on the behavior of Ss involved in the stressful and novel situation of giving birth for the first time, three pregnant albino rats were fed food containing DDT in concentrations of 0,50 , or $100 \mathrm{ppm}$. The maternal behavior of the DDT animals, in the form of nest building, nursing (as measured by time spent nursing and weight of litters), retrieval, and activity level, as compared to the control animal was as predicted by the hypothesis.

In one of the few studies examining the effects of DDT upon behavior, Sobotka (1971) found that low DDT dosages of up to $25 \mathrm{mg} / \mathrm{kg}$ increased motor activity, weakened the ability to habituate to an open-field situation, and tended to reduce passive avoidance. Theoretically, DDT may interfere with the central inhibitory system, retarding the development of habituation to a novel environment. By weakening the cholinergic system and internal inhibitions produced by anxiety, DDT seems to release formerly inhibited motor activity.

Most studies have found that DDT poisoning seems to result in irritability and greater reaction to stimuli (Woodard et al, 1944), as well as in damage to the liver, brain, and other organs (Dale et al, 1963; Goodman \& Gilman, 1970). The irritability and increased reactivity to stimuli are also characteristic of emotional states. However, Khairy (1959), studying problem-solving behavior, speed and pattern of locomotion, and reaction to stress, found that animals exposed to DDT tended to show less emotional reaction to stress, although this tendency was statistically significant only for urination. There were no significant differences in the measures of ambulation, rearing, preening, defecation, squeaking, and feeding.

The following pilot study was undertaken to investigate the effects of a food diet contairing DDT on nest building, nursing, retrieval, and activity level in the stressful and novel situation of giving birth for the first time.

\section{METHOD}

Three 1-week-pregnant albino rats were fed initial dosages of DDT (20\% o' $\mathrm{p}^{\prime}$ and $80 \% \mathrm{p}^{\prime} \mathrm{p}^{\prime}$ form) dissolved in pure peanut oil mixed with milled Noyes pellets as follows: S C, 0 ppm; S 150 , $150 \mathrm{ppm}$; and $\mathrm{S} 700,700 \mathrm{ppm}$. Water was provided ad lib, and Ss were housed in individual $75 \times 75 \times 25 \mathrm{~cm}$ observation cages. The cages consisted of a wooden floor with screen sides and top. The floors were marked off into $15 \times 15 \mathrm{~cm}$ squares.
Three days after DDT feeding began, DDT Ss suffered from acute DDT poisoning, marked by tremors and hyperactivity. In order to prevent loss of the Ss, the DDT mixture was withdrawn and replaced with Noyes pellets for 5 days, until all Ss' behavior had apparently returned to normal.

Dosage of DDT was resumed, with S C getting $0 \mathrm{ppm}, \mathrm{S} 50$ receiving $50 \mathrm{ppm}$, and $S 100$ receiving $100 \mathrm{ppm}$ for 1 week. The day following resumption of DDT diets, strips of paper toweling for nest building and as a measure of maternal behavior were attached to the tops of the cage sides, and the number of strips pulled down daily were counted. Beach (1938), studying the effects of brain lesions on maternal behavior, found that generally the larger the lesion, the more interference with this behavior. Observations were made for $10 \mathrm{~min}$, four times per day at 10:00 a.m., 1:00, 4:00, and 7:00 p.m. for 2 weeks, starting with parturition. The nest building, amount of time spent nursing, daily activity of Ss after parturition, retrieval, and litter weight were observed. Observations were made by three Os, one of whom was aware of the treatment condition of Ss.

\section{RESULTS \\ Nest Building}

S C and S 50 immediately pulled down the strips of paper toweling and made very compact nests 5 days before parturition. S 100 also immediately pulled down strips but made no recognizable nest until the day before parturition.

\section{Nursing}

During the 2 weeks of observation, percentage of time spent nursing for S C was $56 \%$; for S $50,46 \%$; and for S $100,20 \%$.

\section{Retrieval}

Retrieval was measured by E scattering pups around the cage and observing the amount of time necessary for the Ss to return them to their nests (Beach, 1937; Stone, 1938). All Ss retrieved immediately.

\section{Activity Level}

Activity level was measured by the number of squares crossed during the $10 \mathrm{~min}$ observation time. Results were S C, 430 crossings; S 50, 259 crossings; and S 100, 959 crossings. However, we had no recorded measures of activity level before parturition.

\section{Weight of Litters}

The weights of the litters 2 weeks after parturition were as follows: $\mathrm{S} \mathrm{C}$ (five pups), $251.75 \mathrm{~g}$, mean weight $=50.35 \mathrm{~g} ; \mathrm{S} 50$ (nine pups), $260 \mathrm{~g}$, mean weight $=29 \mathrm{~g}$; and $\mathrm{S} 100$ (six pups), $192.5 \mathrm{~g}$, mean weight $=32.1 \mathrm{~g}$. The difference in litter size may account for the weight of S 50's pups and the low activity level of S 50 . 


\section{DISCUSSION}

Results supported the hypothesis, with DDT animals showing significantly less nursing behavior than the control animal and the high-DDT animal exhibiting poor nest building behavior and significantly greater activity level than the low-DDT and control animals. The average weights of the litters at 2 weeks of age would also seem to bear out the nursing results. However, as noted, the difference in S 50's litter weight and activity level may have resulted because the litter size was not equalized.

The findings were interpreted as providing support for the hypothesis that DDT interferes with the development of habituation to a new environment, i.e., giving birth for the first time, and that it leads to increased motor activity and, thereby, has a detrimental effect on maternal behavior. Of course, additional research must be undertaken to corroborate these conclusions. A larger study is currently underway using equal litters and including the measurement of preparturition activity level and other measures of maternal behavior.

\section{REFERENCES}

Beach, F. A. The neural basis of innate behavior: I. Relative effects of cortical lesions upon the maternal behavior pattern in the rat. Journal of Comparative Psychology, 1937, 24, 393-440.

Beach, F. A. The neural basis of innate behavior: II. Relative effects of partial decortication in adulthood and infancy upon the maternal behavior of the primiparous rat. Journal of Genetic Psychology, 1938, 53, 109-148.

Dale, W. E., Gaines, T. B., Hayes, W. J., Jr., \& Pearce, G. W. Poisoning by DDT: Relation between clinical signs and contraction in rat brain. Science, $1963,142,1474-1476$.

Goodman, L. S., \& Gilman, A. The pharmacological basis of therapeutics. New York: McMillan, 1970.

Khairy, $M$. Changes in behavior associated with a nervous system poison (DDT). Quarterly Journal of Experimental Psychology, $1959,11,84-91$

Sobotka, T. J. Behavioral effects of low doses of DDT. Proceedings of the Society for Experimental Biology \& Medicine, 1971, 137, 952-955.

Stone, C. P. Effects of cortical destruction on reproductive behavior and maze learning in albino rats. Journal of Comparative Psychology, 1938, 26, 217-236.

Woodard, G., Nelson, A., \& Calvery, H. Acute and subacute toxicity of DDT $[2,2,-b$ is (p-chloropheny 1$)-1,1,1$ trichloroethane] to laboratory animals. Journal of Pharmacology, 1944, 82, 152-158.

(Received for publication December 20, 1972.) 